\title{
Characteristics of assessment and treatment in Benign Paroxysmal Positional Vertigo (BPPV)
}

\author{
Laura Power ${ }^{\mathrm{a}, \mathrm{b}, *}$, Katherine Murray ${ }^{\mathrm{b}}$ and David J. Szmulewicz ${ }^{\mathrm{a}, \mathrm{c}}$ \\ ${ }^{a}$ Balance Disorders and Ataxia Service, Royal Victorian Eye and Ear Hospital, \\ East Melbourne, VIC, Australia \\ ${ }^{\mathrm{b}}$ Dizzy Day Clinics, Burnley, VIC, Australia \\ ${ }^{\mathrm{c} C e r e b e l l a r}$ Ataxia Clinic, Neuroscience Department, Alfred Health/Monash University, \\ Melbourne, VIC, Australia
}

\begin{abstract}
.
QUESTION: Benign Paroxysmal Positional Vertigo (BPPV) is the most common cause of dizziness presenting to specialist vestibular centres and accounts for approximately $20-30 \%$ of referrals to these clinics. In spite of the amount of clinical knowledge surrounding its diagnosis and management, the treatment of BPPV remains challenging for even the most experienced clinicians. This study outlines the incidence of BPPV in a specialised vestibular physiotherapy clinics and discusses the various nuances encountered during assessment and treatment of BPPV.

DESIGN: Observational Study

PARTICIPANTS: 314 patients with various forms of Benign Paroxysmal Positional Vertigo (BPPV)

INTERVENTION: Canalith repositioning manoeuvres (CRP) for posterior canal (PC) or horizontal canal (HC) BPPV depending on the canal and variant of BPPV.

OUTCOME MEASURES: Negative Dix-Hallpike (DHP) or Supine roll test (SRT) examination.

RESULTS: In 91\% of cases, PC BPPV was effectively treated in 2 manoeuvres or less. Similarly, $88 \%$ of HC BPPV presentations were effectively managed with 2 treatments. Bilateral PC, multiple canal or canal conversions required a greater number of treatments. There was no noticeable difference in treatment outcomes for patients who had nystagmus and symptoms during the Epley manoeuvre (EM) versus those who did not have nystagmus and symptoms throughout the EM. Nineteen percent of patients experienced post treatment down-beating nystagmus (DBN) and vertigo or "otolithic crisis" after the first or even the second consecutive EM.

CONCLUSION: Based on the data collected, we make several clinical recommendations for assessment and treatment of BPPV. Firstly, repeated testing and treatment of BPPV within the same session is promoted as a safe and effective approach to the management of BPPV with a low risk of canal conversion. Secondly, vertigo and nystagmus throughout the EM is not indicative of treatment success. Thirdly, clinicians must remain vigilant and mindful of the possibility of post treatment otolithic crisis following the treatment of BPPV. This is to ensure patient safety and to prevent possible injurious falls. Our results challenge several clinical assumptions about the assessment and treatment of BPPV including the utility of certain markers of treatment success; hence influencing the current clinical guidelines and clinical practice and paving the way for future studies of the assessment and management of patients with BPPV.
\end{abstract}

Keywords: Benign Paroxysmal Positional Vertigo (BPPV)

\footnotetext{
${ }^{*}$ Corresponding author: Laura Power, Research Assistant, Royal Victorian Eye and Ear Hospital, Australia. Tel.: +61 0490106548; E-mail: laura_power@live.com.au.
} 


\section{Introduction}

Benign Paroxysmal Positional Vertigo (BPPV) accounts for approximately $20-30 \%$ of referrals to specialised vestibular clinics [51] with an incidence of BPPV as high as 64 per 100,000 [2,15]. The cumulative lifetime incidence is approximately $10 \%$ at 80 years [51], with the peak incidence of BPPV between 50 and 70 years of age [54] and a predilection towards women at $2.2: 1$ when compared to men [33].

BPPV is a mechanical disorder of the peripheral vestibular system, characterised by repeated brief (less than 1 minute) episodes of positional vertigo caused by crystals of calcium carbonate (otoconia) which are (1) dislodged from the utricle and travel into the semicircular canals (canalithasis) [50], or (2) less commonly, adhering to the cupula and rendering it sensitive to gravity (cupulolithasis) [2]. Posterior canal BPPV (PC BPPV) is the most common canal affected, accounting for 80 to $90 \%$ of cases, followed by horizontal canal BPPV (HC BPPV), (5 to $15 \%$ ) and anterior canal BPPV (AC BPPV) being the least common (1 to 2\%) [5, 7, 25]. Diagnosis of this condition is based on the characteristics of the nystagmus elicited by performing the Dix-Hallpike manoeuvre (DHP)[10, 12] or Supine roll test (SRT) [31] which elicits characteristic nystagmus that follows head movement in the plane of the involved semicircular canal, with features of latency and fatigability [50]. The patient's symptoms of vertigo are commonly reproduced during positioning [10], and BPPV is typically responsive to Canalith repositioning procedures (CRP) [47]. However, it is important to note that the latter is not adequate for diagnosis because other conditions may mimic the symptoms of BPPV [4, 11, 38, 52].

The term 'benign' generally implies that a disease or condition is not harmful in its effect, but despite this label in BPPV, the burden of disease experienced by those suffering from BPPV suggests otherwise. Greater than $60 \%$ of patients with BPPV require leave from work [3], and $86 \%$ experience interruption to activities of daily living [51]. Significant economic and health care costs are associated with hospital admissions, secondary to vertiginous episodes, at approximately two billion dollars annually in the USA for the diagnosis of BPPV alone [27]. Falls and falls-related injuries are significantly higher in BPPV patients, than those without BPPV, with up to $38 \%$ experiencing falls as a direct consequence of BPPV, with the incidence of both BPPV and falls increasing with increasing age [26]. The treatment of positional vertigo has been demonstrated to reduce the incidence of falls by $64 \%$ [20]. Although BPPV may resolve spontaneously, without treatment, up to $50 \%$ of cases may take longer than three months to resolve, hence the CRP is the preferred treatment option [22, 29]. Clinicians must also be wary, that structural cerebellar pathology, (including tumours of the cerebellum $[38,39])$, along with more benign vestibular conditions such as vestibular migraine (VM) $[24,43]$ may mimic BPPV, and accounts for up to $20 \%$ of cases of positional vertigo [4].

Despite a comprehensive understanding of the pathophysiology of BPPV, the assessment and treatment of this condition may be challenging for even the most experienced clinicians. There is a multitude of reasons for the difficulties experienced. They include difficulties in positioning patients due to medical comorbidities (e.g. limited cervical range of motion or lower back pain), challenges interpreting the patterns of nystagmus, BPPV affecting the horizontal, anterior, bilateral or multiple canals and refractory or recurrent BPPV [25, 40]. This study outlines the incidence of BPPV in a specialised vestibular physiotherapy clinic and discusses the various nuances encountered during assessment and treatment of BPPV.

\section{Methods}

A prospective study of 314 patients who presented with a history suggestive of BPPV were assessed by physiotherapists specialising in vestibular disorders in a speciality vestibular physiotherapy clinic in Melbourne, Australia. A comprehensive assessment determined the diagnosis of definite BPPV according to the Barany Society diagnostic criteria for BPPV using the DHP or SRT [5, 12]. These patients were included in the study. Patients whose nystagmus did not concur with the diagnostic characteristics of BPPV, including those with vestibular migraine (VM) or central positioning nystagmus (CPN) were excluded from the study. Diagnosis was based on visual observation of the nystagmus (Frenzel goggles or video-oculography were not employed). A successful treatment was defined by the presence of a negative DHP or SRT (i.e. the absence of nystagmus which represents active BPPV) on retesting within the same clinical session.

Patients who were diagnosed with PC BPPV canalithasis were treated with the Epley manoeuvre (EM) $[13,26]$, or in the case of cupulolithasis, 
the Semont manoeuvre[44]. Patients with HC BPPV canalithasis were treated with either the Barbeque roll (BBQ)[41] or Appiani manoeuvre [1, 29], or in the case of HC cupulolithasis, the modified Brandt Daroff (mBD) or the Casani manoeuvre [9, 30] which was then followed by the appropriate canalithasis manoeuvre. In one case of anterior arm HC BPPV, the Gufoni manoeuvre was applied [46]. A combination of treatments was required if the otoconia resided in more than one canal, i.e. bilateral or multiple canal BPPV. If otoconia resided in more than one canal simultaneously, treatment occurred across more than one treatment session, i.e. one canal treated per day. The combinations included 2 cases of ipsilateral PC and $\mathrm{HC}$ BPPV, 1 case of PC and contralateral HC BPPV, and one case of bilateral PC plus left sided AC BPPV. In the case of AC BPPV, the EM was also carried out. Where an initial treatment selection was unsuccessful (i.e. the DHP or SRT remained positive), an alternate CRP could be selected by the clinician. In the case of canal conversion [18], an alternate CRP was selected to treat the affected canal. Patients were treated with a maximum of 3 manoeuvres within 1 session, or until a negative DHP or SRT was obtained, unless contraindicated (treatment selection was based on clinician preference). In line with the clinical practice guidelines for BPPV, no post treatment precautions were provided to patients post management for their BPPV [5].

Data regarding age, gender, aetiology, associated symptoms, duration of symptoms, positional testing characteristics, canal distribution, nystagmus features, treatment outcomes and incidence of nausea and vomiting during repositioning were recorded at the time of the consultation. All patients provided informed consent. This study was approved by the Royal Victorian Eye and Ear Hospital (RVEEH) Human Research Ethics committee (HREC) (HREC Reference Number: 11/994H/16).

\section{Results}

Three hundred and fourteen patients were diagnosed with BPPV. Subject demographic data is presented in Table 1.

At the time of symptom onset, 100\% (314) of patients reported associated symptoms of vertigo, $75 \%$ (235) experienced gait unsteadiness or balance impairment, 55\% (175) experienced nausea, with $10 \%$ (31) also vomiting. Nineteen percent (59) reported a sensation of head "fogginess" and 6\% (18)
Table 1

Patient demographic data

\begin{tabular}{lc}
\hline Demographic & BPPV \\
\hline Age (mean, range, \pm SD, years) & $61.8(27-93) \pm 15.2$ \\
Sex ratio (female $:$ male) & $229(73 \%): 85(27 \%)$ \\
Time from symptom onset & $43(1$ day to 2 years $) \pm 96$ \\
$\quad$ days, range, \pm SD) & \\
History of migraine & $105(33 \%)$ \\
\hline
\end{tabular}

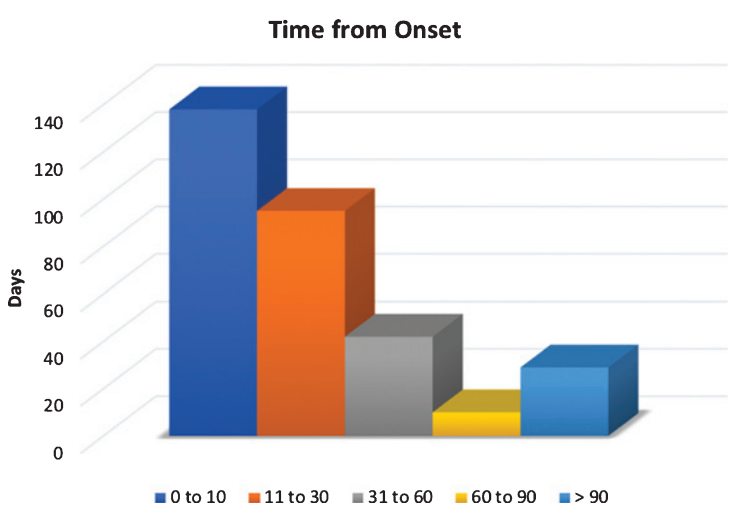

Fig. 1. Time from BPPV symptom onset.

experienced falls as a direct consequence of a vertiginous episode. Seventy percent (219) of patients presented within the first 30 days of symptom onset (Fig. 1) with a median of 42 days.

In some subjects, repeated performance of the DHP was required in order to obtain a positive result. DHP was repeated when the patient history was strongly suggestive of BPPV, and symptoms were relatively close to the time of presentation (e.g. the morning of the assessment). The average number of DHP manoeuvres required to obtain a positive result was 1.15 with a range of 1 to 3 . Thirty patients (10\%) required 2 , whilst 10 patients $(3 \%)$ required 3 DHP manoeuvres before a positive result was obtained. In PC BPPV, DBN during the DHP on the contralateral side occurred in $6 \%$ (15) of patients.

The distribution of BPPV by affected canal is shown in Table 3. Multiple canal BPPV was found in 4 patients (1\%), bilateral PC BPPV in 14 patients $(5 \%)$ and cupulolithasis in 8 patients (3\%). BPPV affected the right ear in 163 patients (52\%), the left side in 133 patients $(42 \%)$. Bilateral PC BPPV was found in in 14 patients $(5 \%)$ and multiple canal BPPV in 4 patients $(1 \%)$. The incidence of multiple canal (4 patients, 11\%) and bilateral posterior canal (2 patients, $6 \%$ ) was higher in the post-traumatic BPPV group (36), with an incidence of $17 \%$, compared to $6 \%$ in the sample of this study. 
Table 2

Aetiology of BPPV

\begin{tabular}{lc}
\hline Aetiology & $\begin{array}{c}\text { Number } \\
\text { (Percentage of cohort) }\end{array}$ \\
\hline Idiopathic BPPV & $272(85 \%)$ \\
Post traumatic BPPV & $36(11 \%)$ \\
Post vestibular neuritis $(\mathrm{VN}) \mathrm{BPPV}$ & $12(4 \%)$ \\
\hline
\end{tabular}

Table 3

Distribution of BPPV by canal(s) affected

\begin{tabular}{|c|c|c|c|c|c|c|}
\hline \multirow{2}{*}{$\begin{array}{l}\text { Affected canal } \\
\text { Canalithasis (Can)/ } \\
\text { Cupulolithasis (Cup) }\end{array}$} & \multicolumn{2}{|c|}{ Posterior } & \multicolumn{2}{|c|}{ Horizontal } & \multicolumn{2}{|c|}{ Anterior } \\
\hline & Can & Cup & Can & Cup & Can & Cup \\
\hline $\mathrm{uml}$ & 248 & 2 & 40 & 6 & 0 & 0 \\
\hline Percentage of patients & & $4 \%$ & & $16 \%$ & & $9 \%$ \\
\hline
\end{tabular}

In PC BPPV, 79\% (197) resolved following one CRP manoeuvre and 91\% (227) after two manoeuvres. The EM was selected as the CRP in 98\% (246) of PC BPPV presentations. If the EM was unsuccessful, or in the case of cupulolithasis, the Semont manoeuvere was performed. In HC BPPV 69\% (31) were resolved after 1 treatment and $88 \%$ (40) with two manoeuvres. In $\mathrm{HC}$ canalithasis, the BBQ roll was performed in $41 \%$ (19) of patients, and in $45 \%$ of patients (21) the Appiani manoeuvre. There were four instances $(4 / 19,21 \%)$ in which the BBQ roll failed to clear the otoconia, and the Appiani manoeuvre was performed resolving the BPPV. In 1 case (5\%) the Appiani failed, and the BBQ roll was then used and resolved the BPPV. Five patients with $\mathrm{HC}$ cupulolithasis underwent several treatments with a median of 2 (range 1-4) treatments being required to obtain symptom resolution.

In those with bilateral PC BPPV 71\% (10) resolved with 2 treatments over 2 treatment sessions. In multiple canal BPPV, 75\% (3) resolved after 3 manoeuvres (over 2 or more treatment sessions). Thirteen of the 314 patients experienced canal conversions (posterior canal to horizontal canal BPPV) and $81 \%$ of those patients resolved in 2 treatments or less. The incidence of canal conversion was 5\% (16). In the post-traumatic group, $83 \%$ (30) of patients were treated in 2 treatments. In the migraine group $94 \%$ (103) resolved in 2 treatments or less. (Fig. 2)

There was minimal difference in treatment outcomes for patients who had nystagmus and vertigo during the EM, versus those that did not (Table 4).

Comparison of type of BPPV and number of treatments required

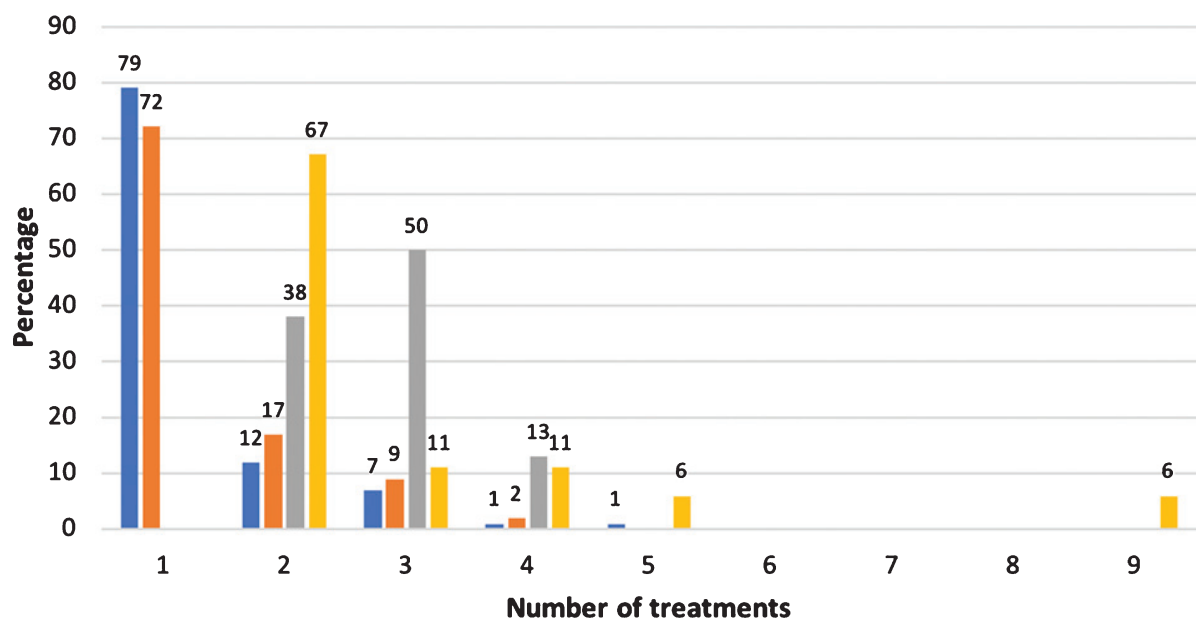

- PC BPPV $\quad$ HC BPPV (Canalithasis) Cupulolithasis Bilateral \& Multiple Canal

Fig. 2. Comparison of type of BPPV and number of treatments required.

Table 4

Vertigo and nystagmus during the Epley manoeuvre (EM)

\begin{tabular}{lcccc}
\hline & 2nd position of EM & 3rd position of EM & Sit up after 1st EM & TLP after 2nd EM \\
\hline Vertigo and nystagmus during the EM & $30 \%(n=75)$ & $35 \%(n=88)$ & $27 \%(n=68)$ & $19 \%(n=45)$ \\
Average number of treatment manoeuvres & 1.41 & 1.28 & 1.21 & 1.32 \\
\hline
\end{tabular}


A post treatment otolithic crisis (thought to represent mass movement of otoconia or an otolithic crisis or catastrophe) occurred in 19\% (48) [30,45] of patients and describes the presence of DBN and vertigo occurring on sitting up after either the first or even the second consecutive EM. Ten of these patients (4\%) experienced simultaneous trunk retropulsion consistent with the otolithic crisis $[30,45]$.

The incidence of nausea and vomiting was also recorded. Ninety-five patients $(30 \%)$ experienced nausea, whilst $22(7 \%)$ vomited during repositioning. In the PC BPPV group, 24\% (60) experienced nausea, whilst $4 \%$ (9) vomited. The incidence of vomiting was higher if the otoconia resided in the HC (11 patients, $25 \%$ ), if there were a past history of migraine (11 patients, $10 \%$ ) or if there was a canal conversion, with half of this latter group experiencing nausea, and 2 patients (12\%) vomiting.

\section{Discussion}

In the sample examined, age and gender predilections were consistent with those reported in the literature $[32,51,54]$. Similarly, a greater incidence of isolated PC BPPV (79\%) when compared to HC BPPV (15\%), and AC BPPV (0\%) reflects what has previously been reported, and so too, the aetiologies (Table 2) $[7,19,25]$. The incidence of bilateral and multiple canal BPPV was higher in the post-traumatic BPPV group $(17 \%, 6 / 36)$, consistent with previous studies [36, 42]. Unlike some previous studies, the subjects in our study presented acutely [21,24]. This discordance may be due to the more lengthy referral processes and waiting times for hospital based tertiary referral centres. The initial assessment undertaken in our study was 43 days from symptom onset (on average) with almost half presenting in the first 10 days of onset. These findings may in part be due to the increased profile of physiotherapy in the assessment and management of BPPV and that patients in the Australian health care system may self-refer to private specialist physiotherapy clinics [53].

Previous studies have suggested that when the patient history is strongly suggestive of BPPV, repeated examination across multiple sessions is required to confirm the diagnosis of BPPV [37]. In our study $13 \%$ of patients (40) required up to three DHP tests to elicit the characteristic nystagmus and confirm a diagnosis of BPPV, highlighting the importance of repeated clinical testing for BPPV within the same session to avoid a false negative DHP or SRT.
In an earlier study, Viirre and Baloh described the importance of repeated DHP within the same session [49]. This practice is more efficient than repeat testing over multiple sessions because it decreases the risks of BPPV such as falls and their sequelae, as well as the cost to the patient and public purse.

Additionally, the findings of our study support retesting for BPPV within the same session following the CRP, to determine the efficacy of the treatment $[23,35][23,35,44,45]$. Many clinicians fear retesting a patient with the DHP in the same session because of the risks of canal conversion or re-entry. This was not the case in our study, where only 5\% (16) underwent a 'canal switch' $[28,49]$ compared to previous studies which reported canal conversion ranging from $6 \%$ $[18]$ to $16 \%[14,47]$. The incidence of vomiting was relatively low in PC BPPV (4\%) but is significantly higher in HC BPPV (25\%), following canal conversions $(12 \%)$, and when there was a past history of migraine (14\%), a finding that has not been reported previously.

Persistent DBN during the DHP test on the contralateral side to the ear affected with PC BPPV has previously been reported to occur in $6-13 \%$ of patients [48], with our study finding $6 \%$ (15) of patients exhibiting this sign. Certain authors have proposed this to be an apogeotropic variant of PC BPPV $[8,48]$. Another alternative theory is the coplanar push-pull mechanism of the $\mathrm{AC}$ of one side and $\mathrm{PC}$ of the other side, resulting in a compensatory DBN on the unaffected side [48]. This DBN typically resolves with treatment of the affected PC, as it did in our experience. This is relevant to the need for any investigation aimed at excluding a structural posterior fossa aetiology [38].

Previous studies have suggested that vertigo and nystagmus during the EM, in particular, in the second position, is required for successful treatment and that absence of this is indicative of treatment failure $[17,22,34]$. Our study found that the incidence of vertigo and nystagmus throughout the EM was not particularly high (19-35\%, Table 5) nor predictive of a successful treatment outcome. A negative repeat DHP or SRT remains the only highly predictive indicator of treatment success $[35,45]$.

In the current study, $90 \%$ of patients were treated effectively with two or less treatment manoeuvres. Bilateral PC BPPV, multiple canal BPPV, cupulolithasis required a greater number of treatment manoeuvres to obtain symptom resolution, which is consistent with past studies [36, 40]. If a patient fails to respond to a modest number of repositioning 
manoeuvres, further investigation is recommended in order to exclude central causes for the presentation, such as cerebellar structural pathology [38, 39]. The number of treatments manoeuvres required in the post-traumatic group was not significantly higher than those in the idiopathic group (unless the otoconia resided in multiple canals simultaneously). In our study $83 \%$ of patients in the post-traumatic BPPV group responded to repositioning in less than 2 treatments compared to $61 \%$ in the literature [16]. Our findings concur with a previous study which showed performing more than one treatment in a single session to obtain a negative outcome, rather than repeated EM over concurrent sessions is effective [23, 44].

When performing repeated EMs within the same session, clinicians must be mindful of the possibility of a second post treatment otolithic crisis [30, 33]. When patients sit up from the second consecutive Epley manoeuvre, irrespective of whether the DHP is negative, $19 \%$ of patients may experience intense but brief vertigo, often perceived as a falling sensation, along with DBN, previously described as similar to the crisis of Turmarkin [30,33]. A smaller percentage of patients (4\% experienced trunk retropulsion, as described previously [6]. We strongly recommend that clinicians be vigilant and support the patient's trunk during the sitting up phase of the EM in the event that they experience truncal retropulsion and/or anteropulsion.

The limitations of this study were: Firstly, the relatively short duration of data collection (10 months) which prevented a comprehensive analysis of BPPV recurrence rates. Secondly, this was a descriptive and observational study of BPPV management in the "real world" of clinical practice at a tertiary centre. We acknowledge controlled trials are required to compare the efficacy of different treatments to determine whether early intervention enhances patient outcomes in patients with BPPV.

\section{Conclusion}

This study offers insight into the 'real world' of BPPV management. Hitherto, similar studies have been performed in tertiary referral centres where access limitations have precluded the data collection of acute BPPV assessment and management.

Based on our data, we recommend when if the patient history is suggestive of BPPV, repeated positional testing within the same session be conducted to confirm or refute the diagnosis of BPPV. Repeated testing and treatment within the same session is a safe and more efficient approach to treatment, with a low risk of canal conversion. In PC BPPV, vertigo and nystagmus during the EM are not indicative of treatment success. Performing the DHP and SRT (as relevant) remain the only indicators of treatment outcome. We strongly recommend, if patients do not respond promptly to CRP, further investigation is mandatory to exclude cerebellar or brainstem structural pathology.

\section{Ethics approval}

This study was approved by the Royal Victorian Eye and Ear Hospital (RVEEH) Human Research Ethics committee (HREC) (HREC Reference Number: $11 / 994 \mathrm{H} / 16)$.

\section{Acknowledgments}

We wish to acknowledge the team members at Dizzy Day Clinics for their efforts in data collection on this project: Arimbi Winoto, Belinda Reid, Amy Gillon, Mandy Lenwood and Marianna Harrison.

\section{References}

[1] G.C. Appiani, G. Catania and M. Gargliardi, A libratory maneuver for the treatment of horizontal canal paroxysmal positional vertigo, Otol Neurotol 22 (2001), 66-9.

[2] R.W. Baloh, Q. Yue, K.M. Jacobson and V. Honrubia, Persistent direction-changing positional nystagmus: Another variant of benign positional nystagmus? Neurology 45(7) (1995), 1297-1301.

[3] H. Benecke, S. Agus, D. Kuessner, G. Goodall and M. Strupp, The burden and impact of vertigo: Findings from the REVERT patient registry, Front Neurol 4 (2013), 7.

[4] P. Bertholon, S. Tringali, M.B. Faye, J.C. Antoine and C. Martin, Prospective study of positional nystagmus in 100 consecutive patients, Annals of Otology, Rhinology \& Laryngology 115(8) (2006), 587-594.

[5] N. Bhattacharyya, S.P. Gubbels, S.R. Schwartz, J.A. Edlow, H. El-Kashlan, T. Fife, J.M. Holmberg, K. Mahoney, D.B. Hollingsworth, R. Roberts, M.D. Seidman, R.W. Steiner, B.T. Do, C.C. Voelker, R.W. Waguespack and M.D. Corrigan, Clinical Practice Guideline: Benign Paroxysmal Positional Vertigo (Update). Otolaryngol Head Neck Surg 156(3_suppl) (2017), S1-S47.

[6] B. Büki, L. Simon, S. Garab, Y.W. Lundberg, H. Jünger and D. Straumann, Sitting-up vertigo and trunk retropulsion in patients with benign positional vertigo but without positional nystagmus, Journal of Neurology, Neurosurgery \& Psychiatry 82(1) (2011), 98-104. 
[7] B.O. Cakir, I. Ercan, Z.A. Cakir, S. Civelek, I. Sayin and S. Turgut, What is the true incidence of horizontal semicircular canal benign paroxysmal positional vertigo? Otolaryngol Head Neck Surg 134(3) (2006) 451-4.

[8] S. Carmona, G. Zalazar, R. Weisnchelbaum, G. Grinstein, H. Breinbauer and G.A. Libonati, Downbeating nystagmus in Benign Paroxysmal Positional Vertigo: An apogeotrophic variant of posterior semicircular canal, Current Opinion in Neurological Science 1(6) (2017), 301-305.

[9] A. Casani, G. Vannucci, B. Fattori, S. Berrettini, The treatment of horizontal canal positional vertigo: Our experience in 66 cases, Laryngoscope 112 (2002), 172-8.

[10] M.B. Chang, A.P. Bath and J.A. Rutka, Are all atypical positional nystagmus patterns reflective of central pathology? The Journal Of Otolaryngology 30(5) (2001), 280-282.

[11] B.H. Cho, S.H. Kim, S.S. Kim, Y.J. Choi and S.H. Lee, Central positional nystagmus associated with cerebellar tumors: Clinical and topographical analysis, J Neurol Sci 373 (2017), 147-151.

[12] M.R. Dix and C.S. Hallpike, The Pathology, symptomology and diagnoses of certain common disorders of the vestibular system, Proc Soc Med 45 (1952), 341.

[13] J.M. Epley, The canalith repositioning procedure for treatment of benign paroxysmal positional vertigo, Otolaryngol Head Neck Surg 107 (1992), 399-404.

[14] C.A. Foster, K. Zaccaro, D. Strong, Canal Conversion and Reentry: A Risk of Dix-Hallpike During Canalith Repositioning Procedures, Otology \& Neurotology 33 (2012), 199-203.

[15] D.A. Froehling, M.D. Silverstien, D.N. Mohr, C.W. Beatty, K.P. Offord and D.J. Ballard, Benign positional vertigo: Incidence and prognosis in a population-based study in Olmsted County, Minnesota, Mayo Clin Proc 66 (1991), 596-601.

[16] C.R. Gordon, R. Levite and V. Joffe, Is Posttraumatic Benign Paroxysmal Positional Vertigo Different From the Idiopathic Form? Arch Neurol 61(10) (2004), 1590-1593.

[17] J.O. Helminski, Effectiveness of the Canalith Repositioning Procedure in the Treatment of Benign Paroxysmal Positional Vertigo, Physical Therapy 94(10) (2014), 1373-1382.

[18] S. Herdman and R.J. Tusa, Complications of the canalith repositioning procedure, Archives of Otolaryngology Head \& Neck Surgery (3) (1996), 281.

[19] S.J. Herdman and R.A. Clendaniel, Vestibular Rehabilitation. 4 ed. 2014, Philidelphia: F.A. Davis Company.

[20] K. Jumani and J. Powell, Benign paroxysmal positional vertigo: Management and its impact on falls, Annals of Otology, Rhinology \& Laryngology 126(8) (2017), 602-605.

[21] J.-S. Kim, Clinical characteristics of benign paroxysmal positional vertigo in Korea: A multicenter study, Journal of Korean Medical Science 21(3) (2006), 539-543.

[22] J.S. Kim, Predicting a successful treatment in posterior canal benign paroxysmal positional vertigo, Neurology 68 (2007), 1219-1222.

[23] G.P. Korn, R.S. Dorigueto, M. Ganança and H. Caovilla, Repeated Epley's maneuver in the same session in benign positional paroxysmal vertigo, Brazilian Journal of Otorhinolaryngology 73 (2007), 533-539.

[24] S. Korres and D. Balatsouras, Diagnostic, pathophysiologic, and therapeutic aspects of benign paroxysmal positional vertigo, Otolaryngology - Head \& Neck Surgery (4) (2004), 438.

[25] S. Korres, D.G. Balatsouras, A. Kaberos, C. Economou, D. Kandiloros and E. Ferekidis, Occurence of semicircular canal involvement in benign paroxysmal positional vertigo, Otology \& Neurotology 23 (2002), 926-932.
[26] J. Lawson, D. Bamiou, H.S. Cohen and J. Newton, Positional vertigo in a falls service. Age \& Ageing, 37(5) (2008), 585-589.

[27] J.C. Li, Cost-effective management of benign positional vertigo using canalith repositioning, Mosby: United States, 2000 , p. 334.

[28] G.C. Lin, G.J. Basura, H.T. Wong and K.D. Heidenreich, Canal switch after canalith repositioning procedure for benign paroxysmal positional vertigo, Laryngoscope $\mathbf{1 2 2}$ (2012), 2076-2078.

[29] S. Lynn, A. Pool and D. Rose, Randomized trial of the canalith repositioning procedure, Otolaryngol Head Neck Surg 113 (1995), 712.

[30] E.T. Maranhao, S.L. Whitney and P. Maranhao-Filho, Tumarkin-like phenomenon as a sign of therapeutic success in benign paroxysmal positional vertigo, 2018, pp. 534-538.

[31] J.A. McClure, Horizontal canal BPV, The Journal Of Otolaryngology 14(1) (1985), 30-35.

[32] S. Moon, J. Kim, B.K. Kim, J. Kim, H. Lee, S. Son, K. Kim, G. Han, C. Rhee and W. Lee, Clinical characteristics of benign paroxysmal positional vertigo in korea: A multicentre study, J Korean Med Sci 21 (2005), 539-543.

[33] H. Neuhaeusser and T. Lempert, Vertigo: Epidemiologic Aspects, Seminars in Neurology (5) (2009), 473.

[34] H.J. Oh, J.S. Kim, B.I. Han and J.G. Lim, Predicting a successful treatment in posterior canal benign paroxysmal positional vertigo, American Academy of Neurology: United States, 2007, p. 1219.

[35] A. Oliveira, F. Akira Suzuki and L. Boari, Is it important to repeat the positioning maneuver after the treatment for benign paroxysmal positional vertigo? Brazilian Journal of Otorhinolaryngology 81 (2015), 197-201.

[36] V. Pisani, S. Mazzone, R. Di Mauro, P. Giacomini and S. Di Girolamo, A survey of the nature of trauma of post-traumatic benign paroxysmal positional vertigo, International Journal of Audiology 54(5) (2015), 329-333.

[37] L. Pollak, The Importance of Repeated Clinical Examination in Patients With Suspected Benign Paroxysmal Positional Vertigo, Otology \& Neurotology 30(3) (2009), 356-358.

[38] L. Power, K. Murray, K.J. Drummond, N. Trost and D.J. Szmulewicz, Fourth ventricle ependymoma mimicking benign paroxysmal positional vertigo, Neurology 91(7) (2018), 327-328.

[39] L. Power, K. Murray, K. Bulluss, K. Drummond, N. Trost and D. Szmulewicz, Central conditions mimicking Benign Paroxysmal Positional Vertigo (BPPV) - A Case Series, Journal of Neurologic Physical Therapy 2019, [Manuscript Accepted].

[40] L. Power, K. Murray and D. Szmulewicz, Early Experience with a multi-axial whole body positioning system in the treatment of Benign Paroxysmal Positional Vertigo (BPPV), Journal of Clinical Neuroscience, 2018.

[41] K. Sekine, T. Imai, G. Sato, M. Ito and N. Takeda, Natural history of benign paroxysmal positional vertigo and efficacy of Epley and Lempert maneuvers, Otolaryngol Head Neck Surg 135(4) (2006), 529-33.

[42] D. Shim, C. Song, E. Jung, K. Ko, J. Park and M. Song, Benign Paroxysmal Positional Vertigo with Simultaneous Involvement of Multiple Semicircular Canals, Korean Journal of Audiology 18(3) (2014), 126-130.

[43] R.L. Taylor, L. Chen, C. Lechner, S.T. Aw and M.S. Welgampola, Vestibular schwannoma mimicking horizontal cupulolithiasis, 2013, pp. 1170-1173. 
[44] S.P. Teo, Semont manoevere for vertigo assessment, Aust Fam Physician 44(7) (2015), 471-473.

[45] A. Tumarkin, The Otolithic Catastrophe: A New Syndrome, The British Medical Journal (3942) (1936), 175.

[46] E.M. van den Broek, H.J. van der Zaag-Loonen and T.D. Bruintjes, Systematic Review: Efficacy of Gufoni Maneuver for Treatment of Lateral Canal Benign Paroxysmal Positional Vertigo with Geotropic Nystagmus, Otolaryngol Head Neck Surg 150(6) (2014), 933-8.

[47] J.G. van Duijn, L.M. Isfordink, J.A. Nij Bijvank, C.W. Stapper, A.J. van Vuren, I. Wegner, M.F. Kortekaas and W. Grolman, Rapid systematic review of the epley maneuver for treating posterior canal benign paroxysmal positional vertigo, Otolaryngol Head Neck Surg 150(6) (2014), 92532.

[48] P. Vannucchi, R. Pecci and B. Giannoni, Posterior Semicircular Canal Benign Paroxysmal Positional Vertigo Presenting with Torsional Downbeating Nystagmus: An Apogeotropic Variant, International Journal of Otolaryngology, 2012, 1-9.

[49] E. Viirre, I. Purcell, R.W. Baloh, J.F. Damrose and H. Djalilian, The Dix-Hallpike Test and The Canalith Repositioning Maneuver, LARYNGOSCOPE: United States, 2005, p. 184.
[50] M. von Brevern, P. Bertholon, T. Brandt, T. Fife, T. Imai, D. Nuti and D. Newman-Toker, Benign paroxysmal positional vertigo: Diagnostic criteria Consensus document of the committee for the classification of vestibular disorders of the bárány society, Acta Otorrinolaringologica Espanola 68(6) (2017), 349-360.

[51] M. von Brevern, A. Radtke, F. Lezius, M. Feldmann, T. Ziese, T. Lempert and H. Neuhauser, Epidemiology of benign paroxysmal positional vertigo: A population based study, J Neurol Neurosurg Psychiatry 78(7) (2007), 710-5.

[52] M. Von Brevern, A. Radtke, A. Clarke and T. Lempert, Migrainous vertigo presenting as episodic positional vertigo, Neurology 62(3) (2004).

[53] V.S. Webster, L.K. Holdsworth, A.K. McFadyen and H. Little, Self-referral, access and physiotherapy: Patients knowledge and attitudes - results of a national trial, Physiotherapy 94(2) (2008), 141-149.

[54] N. West, S. Hansen, M.N. Moller, S.L. Bloch and M. Klokker, Repositioning chairs in benign paroxysmal positional vertigo: Implications and clinical outcome, Eur Arch Otorhinolaryngol 273(3) (2016), 573-80. 